\title{
Predicting an accelerated lung function decline in smokers: is there a proper threshold?
}

\section{To the Editor:}

We read, with very much interest, the publication by AKKERMANs et al. [1] in the European Respiratory Journal. In this study they reanalysed data from the first Lung Health Study by forming four groups, based on the forced expiratory volume in $1 \mathrm{~s}$ (FEV1)/forced vital capacity (FVC) $<0.70$ or $<5$ th percentile, according to the LMS (lambda, mu, sigma) approach. However, we do have some remarks concerning the validity of their analysis and propose a means of reinterpretation of their outcome.

AKKERMANs et al. [1] concluded that the decline of the post bronchodilator FEV1 in the group fixed ${ }^{+} / \mathrm{LMS}^{-}$ $\left(\mathrm{FEV} 1 / \mathrm{FVC}<0.70\right.$ and $>5$ th percentile) was significantly lower compared to the group fixed ${ }^{+} / \mathrm{LMS}^{+}\left(\mathrm{FEV}_{1} /\right.$ FVC $<0.70$ and $<5$ th percentile). The mean \pm SD decline was $43.8 \pm 50.0 \mathrm{~mL} \cdot$ year $^{-1}$ versus $53.5 \pm 51.5$ $\mathrm{mL} \cdot$ year $^{-1}$, respectively, and thus one recommended the use of the 5 th percentile threshold to define the presence of chronic obstructive pulmonary disease (COPD).

Interestingly, this conclusion was reached after they excluded, in total, 1842 subjects from the initial 5887 subjects. Therefore, the question arises: what is the consequence of such a vast post hoc exclusion on the estimates of the FEV1 decline in the two groups?

The majority of subjects ( $\mathrm{n}=1276$ ) were excluded as they showed "an unstable classification" and one can ask oneself what the FEV1 decline was in those excluded subjects. Some were reported to have an improved lung function over time and some were reported to have become more obstructive. Data, on whether this exclusion was "randomised" over the four groups formed, is lacking. The text of the publication does not give detailed information on the reasons as to why the authors chose to follow this path. Anyway, one must realise that it is highly likely that the groups, thus formed, suffer from selection bias and the outcome of the analysis is, therefore, also subject to some degree of bias. This selection bias is illustrated in table 1 of the article [1] where it is shown that the excluded subjects had a better FEV1 and FEV1/FVC, despite the same amounts of pack-years.

Of the remaining 566 excluded subjects, a number were excluded based on "missing at least one follow-up spirometry". Now, the authors used the classical statistical approach for longitudinal studies, a random intercept and random slope analysis (mixed procedure in SAS (SAS software 9.2; SAS Institute Inc., Cary, NC, USA)). This type of analysis is characterised by the fact that such missing data is not detrimental to the validity of the outcome. The number of observations within a subject does not appear to be equal for all. It seems that the authors excluded too many subjects than was strictly necessary.

Even when the exclusion of subjects does not lead to bias in the outcome, the conclusion that the FEV1/FVC $<5$ th percentile threshold is to be favoured over the FEV1/FVC $<0.70$ has to be interpreted with caution. If indeed, the decline in $<5$ th percentile group is steeper, it does not mean that in the FEV1/FVC $<0.70$ but $<5$ th percentile group decline is absent. The mean decline differed by a small $10 \mathrm{~mL} \cdot \mathrm{year}^{-1}$, but in both groups is it steeper than expected in healthy subjects (estimated to be $\sim 27 \mathrm{~mL} \cdot \mathrm{year}^{-1}$ for the FEV1, according to the European Respiratory Society reference equations [2]). When the FEV1 decline in the FEV1/FVC $<0.70$ but $>5$ th percentile group is normally distributed with a mean \pm SD of $43.8 \pm$ $50.0 \mathrm{~mL} \cdot$ year $^{-1}$, it is evident that in this group some very steeply declining subjects are present.

AKKERMANS et al. [1] correctly state that a rapid lung function decline is pivotal [3]. When a rapidly declining subject still shows a post bronchodilator FEV1/FVC $\geqslant 5$ th percentile, that subject receives a nonCOPD label and has to wait for that crossing of the 5th percentile threshold before the COPD label is imposed. This means a loss of time, while it is clear that such a rapid-declining subject is truly diseased, those subjects with an initial (very) high FEV1 (e.g. a starting value at the 95th percentile) must "wait" a long time before they receive a proper label, i.e. while their FEV1 deteriorates.

In conclusion, in our view the discussion on which threshold to follow/choose should be replaced by a debate on how to locate rapidly declining subjects with a whether or not they hover still above, at, or already below whatever threshold. 

http://ow.ly/pPIlU

Firdaus A.A. Mohamed Hoesein ${ }^{1}$ and Pieter Zanen ${ }^{2}$

${ }^{1}$ Dept of Radiology, University Medical Center Utrecht, Utrecht, and ${ }^{2}$ Division of Heart and Lungs, Dept of Respiratory Medicine, University Medical Center Utrecht, Utrecht, The Netherlands.

Correspondence: F.A.A. Mohamed Hoesein, University Medical Center Utrecht, HP. F.02.333, P.O. Box 85500, 3508 GA Utrecht, The Netherlands. E-mail: fmohamedhoesein@gmail.com

Received: May 212013 | Accepted: May 242013

Conflict of interest: None declared.

\section{References}

1 Akkermans RP, Biermans M, Robberts B, et al. COPD prognosis in relation to diagnostic criteria for airflow obstruction in smokers. Eur Respir J 2014; 43: 54-63.

2 Quanjer PH, Tammeling GJ, Cotes JE, et al. Lung volumes and forced ventilatory flows. Eur Respir J 1993; 6: Suppl. $16,5-40$.

3 Vestbo J, Edwards LD, Scanlon PD, et al. Changes in forced expiratory volume in 1 second over time in COPD. N Engl J Med 2011; 365: 1184-1192.

$$
\text { Eur Respir J 2014; 43: 307-308 | DOI: 10.1183/09031936.00086713 | Copyright @eERS } 2014
$$

\section{From the authors:}

We thank F.A.A. Mohamed Hoesein and P. Zanen for their interest in our work [1]. In our study, we performed secondary analyses on data from the Lung Health Study (LHS) [2]. We subdivided the LHS study population into four categories based on the presence or absence of airflow obstruction as defined the fixed forced expiratory volume in $1 \mathrm{~s}$ (FEV1)/forced vital capacity (FVC) $<0.70$ cut-off point and by the lower fifth percentile using the LMS (lambda, mu, sigma) method definitions. The LMS method accounts for sex- and age-specific predicted values $(\mathrm{mu})$, and adjusts for nonuniform dispersion (sigma) and skewness of the lung function values distribution (lambda). The original LHS study population consisted of 5887 smokers aged 35-60years. We excluded 1842 subjects because 1276 subjects were in different categories based on the fixed and LMS definitions for airflow obstruction during their baseline and first annual visits, and 566 subjects had missing spirometry results during either their baseline visit or their first annual follow-up visit. This means that for these 566 subjects we did not have the two measurements required to either confirm or refute their consistent classification in a particular category, which was the only reason to exclude them. We agree with F.A.A. Mohamed Hoesein and P. Zanen that missing data (if completely at random) in mixed-models analysis do not influence the validity of the outcome and that subjects with missing data can be included in the analysis. But as explained above, the reason to exclude the 566 subjects from our analysis was not missing data, but uncertainty about their classification.

Because the goal of our study was to compare clearly defined and consistent groups of subjects based on the fixed and LMS definitions for airflow obstruction, we only included those subjects who did not shift between categories during their baseline and first annual follow-up visit (table 1). As a consequence, 1276 (24\%) subjects were excluded from the analysis. This finding shows that a one-off spirometry test does not seem to be sufficient to determine airflow obstruction in a substantial proportion of subjects and suggests that a chronic obstructive pulmonary disease (COPD) diagnosis should not be based on a single spirometry test. However, excluding these subjects clearly comes at the cost of generalisability. Therefore, as stated in the Discussion section of the paper [1], our analysis should be seen as a "proof of concept" and illustrating that, when diagnosing COPD, it seems more appropriate to use sex- and age-specific cut-off points for the FEV1/FVC ratio than it is to use a "one size fits all" fixed (0.70) cut-off point.

The mean decline in both groups is indeed steeper than one would expect in healthy subjects, but this study included only heavy smokers (with a mean \pm SD cumulative cigarette smoke exposure of $40.1 \pm 18.3$ packyears and $31.1 \pm 12.6$ cigarettes smoked per day). We see this as an explanation for the relatively strong annual FEV1 decline of $43.8 \mathrm{~mL} \cdot$ year $^{-1}$. A systematic review by LEE and FRY [3] showed an annual decline of $42.8 \mathrm{~mL} \cdot$ year $^{-1}$ for continuing smokers, which is comparable with the decline we observed in our population. LEE and FRY [3] also showed that continuing smokers have a decline over $10 \mathrm{~mL} \cdot \mathrm{year}^{-1}$ greater than never-smokers, ex-smokers or quitters. Despite the relatively steep overall decline, we still found a 